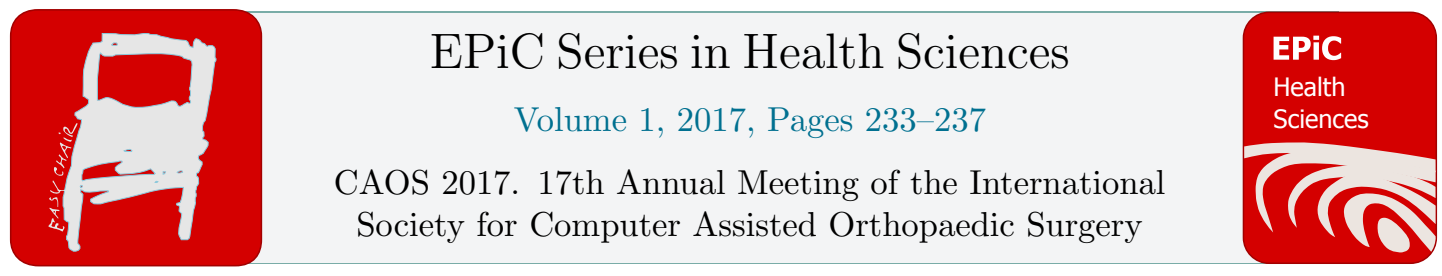

\title{
Morpho-Functional Analysis of Knee Implant Design for Total Knee Arthroplasty on the Example of the Trochlear Groove Orientation
}

\author{
Malte Asseln ${ }^{1 *}$, Mark Verjans ${ }^{1}$, Darius Zanke ${ }^{1}$ and Klaus Radermacher ${ }^{1}$ \\ ${ }^{1 *}$ Chair of Medical Engineering, Helmholtz Institute for Biomedical Engineering, RWTH Aachen \\ University, Aachen, 52074, Germany \\ asseln@hia.rwth-aachen.de
}

\begin{abstract}
Total knee arthroplasty (TKA) is widely accepted as a successful surgical intervention to treat osteoarthritis and other degenerative diseases of the knee. However, present statistics on limited survivorship and patient-satisfaction emphasize the need for an optimal endoprosthetic care. Although, the implant design is directly associated with the clinical outcome comprehensive knowledge on the complex relationship between implant design (morphology) and function is still lacking.

The goal of this study was to experimentally analyze the relationship between the trochlear groove design of the femoral component (iTotal CR, ConforMIS, Inc., Bedford, MA, USA) and kinematics in an in vitro test setup based on rapid prototyping of polymer-based replica knee implants.

The orientation of the trochlear groove was modified in five different variations in a self-developed computational framework. On the basis of the reference design, one was medially tilted $\left(-2^{\circ}\right)$ and four were laterally tilted $\left(+2^{\circ},+4^{\circ},+6^{\circ},+8^{\circ}\right)$. For manufacturing, we used rapid prototyping to produce synthetic replicates made of Acrylnitril-Butadien-Styrol (ABS) and subsequent post-processing with acetone vapor. The morpho-functional analysis of the replicates was performed in our experimental knee simulator. Tibiofemoral and patellofemoral kinematics were recorded with an optical tracking system during a semi-active flexion/extension $\left(\sim 10^{\circ}\right.$ to $\left.90^{\circ}\right)$ motion.

Looking at the results, the patellofemoral kinematics, especially the medial/lateral translation and internal/external rotation were mainly affected. During low flexion, the patella had a more laterally position relative to the femur with increasing lateral trochlear orientation. The internal/external rotation initially differentiated and converged with flexion. Regarding the tibiofemoral kinematics, only the tibial internal/external rotation showed notable differences between the modified replica implants.

We presented a workflow for an experimental morpho-functional analysis of the knee and demonstrated its feasibility on the example of the trochlear groove orientation
\end{abstract}


Morpho-Functional Analysis of Knee Implant Design for Total Knee Arthroplasty ... M. Asseln et al.

which might be used in the future for comprehensive implant design parameter optimization, especially in terms of image based computer assisted patient-specific implants.

\section{Introduction}

Total knee arthroplasty (TKA) is widely accepted as a successful surgical intervention to treat osteoarthritis and other degenerative diseases of the knee. However, present statistics on limited survivorship and patient-satisfaction emphasize the need for an optimal endoprosthetic care. The natural joint surfaces are replaced by artificial components with the aim to relief pain and restore function to improve health-related quality of life. Although, the implant design is directly associated with the clinical outcome (Bonnin et al., 2013; Chung et al., 2015) comprehensive knowledge on the complex relationship between implant design (morphology) and function is still lacking. This might be even more relevant in image based computer assisted patient-specific implant design with the potential to find an individual morpho-functional optimum.

The design of the tibiofemoral joint (conformity) is technically inspired to minimize contact pressures and consequently wear. In literature, e.g. a high frontal but moderate sagittal conformity is suggested to make a compromise between the conflicting contact mechanics and kinematics (Sathasivam and Walker, 1999). Although the impact of conformity on TKA performance represents an important general design principle (Ardestani et al., 2015) the patellofemoral joint is less constrained and considered in literature, wherefore it is the central aspect of this study.

The trochlear groove is a key factor for the guidance of the patella (Steinbruck et al., 2011). Changes in the morphology directly influence its movement on the distal femoral surface during knee flexion/extension. A medial/lateral shift of the patella causes a change in the pulling direction of the M. quadriceps which might affect the internal/external rotation of the tibia (Kienapfel et al., 2003). Extreme lateralization might even result in sub-/luxation.

The goal of this study was to experimentally analyse the relationship between the trochlear groove design of the femoral component and kinematics in an in vitro test setup (knee simulator) based on previous approaches by our group for parameterization of the knee shape and rapid prototyping of replica knee implants (Asseln et al., 2016; Verjans et al., 2016).

\section{Materials and Methods}

Initially, a CAD file of the femoral component (iTotal, ConforMIS, Inc., Bedford, MA, USA) has been parameterized in a self-developed computational framework in MATLAB (Asseln et al., 2016). Subsequently, the orientation of the trochlear groove of the prosthesis was modified in five different variations. On the basis of the reference design, one was medially tilted $\left(-2^{\circ}\right)$ and four were laterally tilted $\left(+2^{\circ},+4^{\circ},+6^{\circ},+8^{\circ}\right)$ (Figure 1).

Based on the manipulated surface models generated by the parameter model, the variants were transferred to volume models for manufacturing. However, the traditional producing of implants made of $\mathrm{CoCr}$ and UHMWPE is cost and time intensive, even though the requirements for the application in in vitro tests, especially in terms of durability and biocompatibility, are lower in comparison to in vivo conditions. For this reasons, we used rapid prototyping to produce synthetic replicates made of Acrylnitril-Butadien-Styrol (ABS) and subsequent post-processing with acetone vapor. The workflow has been successfully evaluated (Verjans et al., 2016).

The morpho-functional analysis of the replicates was performed in our experimental knee simulator. Therefore, the femoral replicates and original UHMWPE tibia and patella components 
were mounted in an artificial specimen. The collateral ligaments were represented by elastic rubber bands and cruciate ligaments were neglected. Five muscle strings were approximated by pneumatic muscles. Tibiofemoral and patellofemoral kinematics were recorded with an optical tracking system during a semi-active flexion/extension $\left(\sim 10^{\circ}\right.$ to $\left.90^{\circ}\right)$ motion.

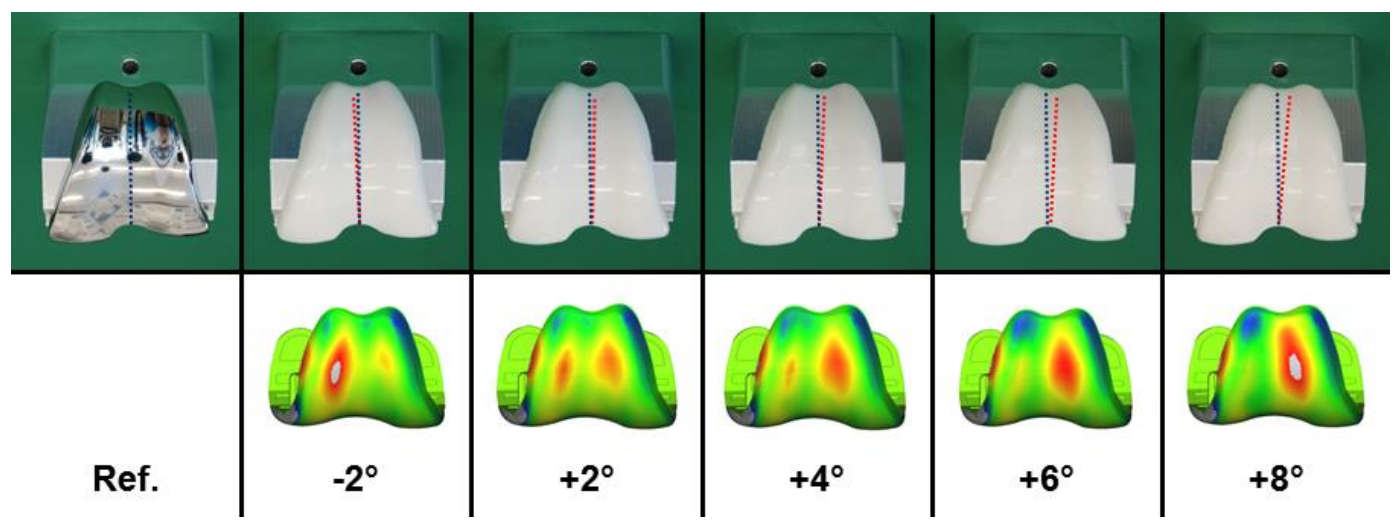

Figure 1: Original femoral component (reference) and five corresponding replica implants with design modifications of the trochlear groove orientation from $-2^{\circ}$ medially to $+8^{\circ}$ laterally

\section{Results}

Looking at the patellofemoral kinematics, especially the medial/lateral translation and internal/external rotation were mainly affected (Figure $2 a)+b)$ ). During low flexion, the patella had a more laterally position relative to the femur with increasing lateral trochlear orientation. During knee flexion, all variations progressed towards the same value. The internal/external rotation initially differentiated and converged with flexion to a $3{ }^{\circ}$ outer rotation. The other kinematics was less influenced.

Regarding the tibiofemoral kinematics, only the tibial internal/external rotation showed notable differences between the modified replica implants (Figure $2 \mathrm{c}$ )). The characteristic course stayed the same, but with raising lateral orientation of the trochlear groove, the overall rotation resulted in a larger external rotation. 
Morpho-Functional Analysis of Knee Implant Design for Total Knee Arthroplasty ... M. Asseln et al.

a)

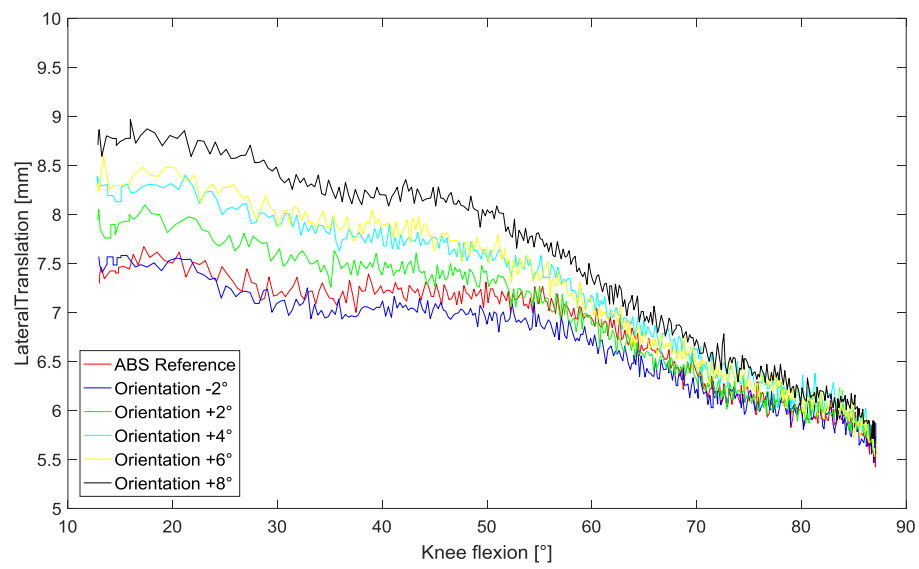

b)

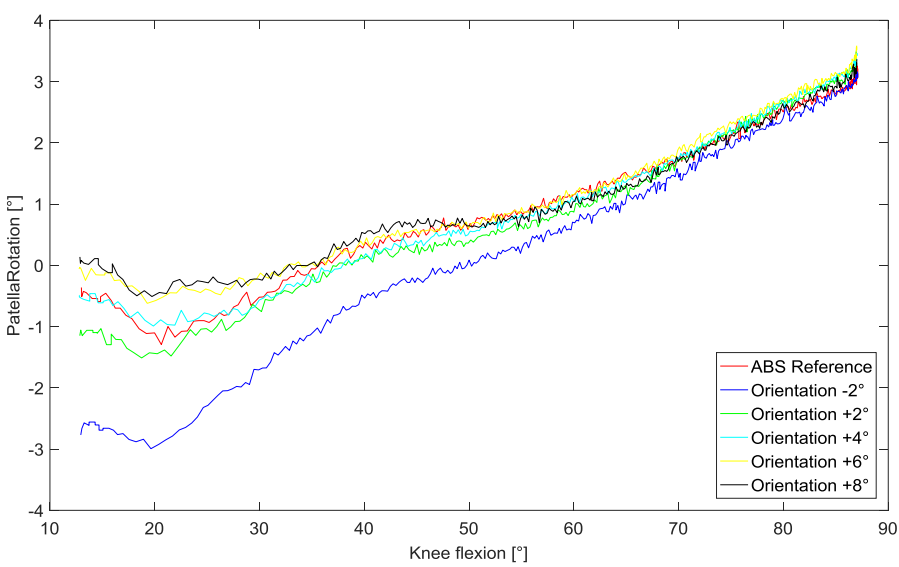

c)

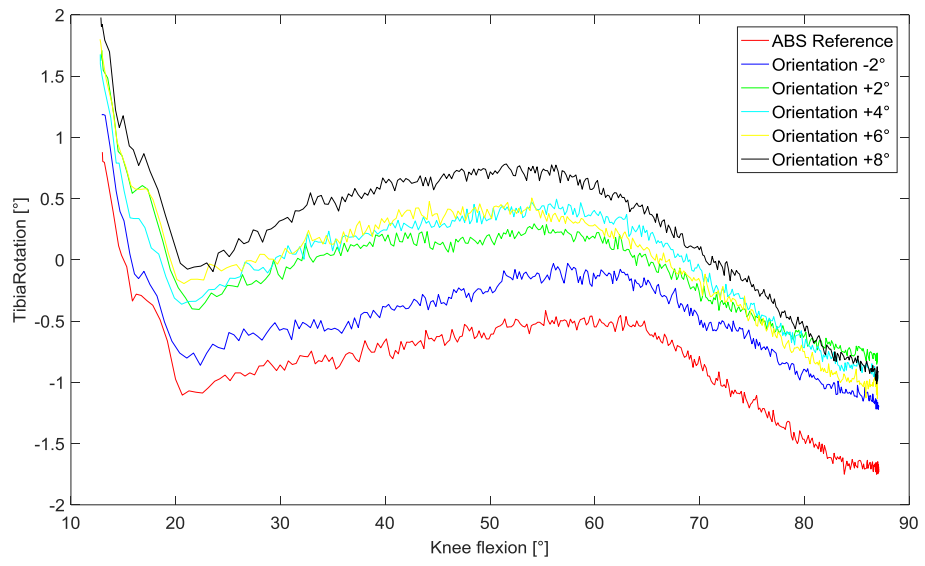

Figure 2: Kinematic results of the in vitro study, a) patellofemoral medial/lateral translation, b) patellofemoral internal/external rotation, c) tibiofemoral internal/external rotation 
Morpho-Functional Analysis of Knee Implant Design for Total Knee Arthroplasty ... M. Asseln et al.

\section{Discussion}

In this study, the orientation of the trochlear groove has been systematically varied and resulting kinematics have been analysed in vitro. As described in the literature, the trochlear groove serves as a guide for the patella during knee flexion. This correlation could be proved by the medial/lateral translation of the patella. A comparison of the variants showed courses which were sorted by a rising lateral orientation with a more lateral located patella on the femur surface in lower flexion.

Additionally, changes in the internal/external tibiofemoral kinematics were observed. A stronger laterally oriented trochlear groove also increased the external rotation of the tibia. Kienapfel et al. found that a medialization of the patella resulted in an internal rotation of the tibia. These findings were in agreement with our observations.

In conclusion, we presented a workflow for an experimental morpho-functional analysis of the knee and demonstrated its feasibility on the example of the trochlear groove orientation which might be used in the future for comprehensive implant design parameter optimization, especially in terms of image based computer assisted patient-specific implants. The approach could be also generalized to address additional joints.

\section{References}

Ardestani, M.M., Moazen, M., Jin, Z., 2015. Contribution of geometric design parameters to knee implant performance: Conflicting impact of conformity on kinematics and contact mechanics. The Knee 22, 217-224.

Asseln, M., Hanisch, C., Al Hares, G., Eschweiler, J., Radermacher, K., 2016. Automatic parameterisation of the distal femur based on 3D surface data: a novel approach for systematic morphological analysis und optimisation. Bone \&amp; Joint Journal Orthopaedic Proceedings Supplement 98-B, 31.

Bonnin, M.P., Schmidt, A., Basiglini, L., Bossard, N., Dantony, E., 2013. Mediolateral oversizing influences pain, function, and flexion after TKA. Knee surgery, sports traumatology, arthroscopy : official journal of the ESSKA 21, 2314-2324.

Chung, B.J., Kang, J.Y., Kang, Y.G., Kim, S.J., Kim, T.K., 2015. Clinical Implications of Femoral Anthropometrical Features for Total Knee Arthroplasty in Koreans. The Journal of arthroplasty 30, 1220-1227.

Kienapfel, H., Springorum, H.-P., Ziegler, A., Klose, K.-J., Georg, C., Griss, P., 2003. Der Einfluss der Femur- und Tibiakomponentenrotation auf das patellofemorale Versagen beim kunstlichen Kniegelenkersatz. Der Orthopade 32, 312-318.

Sathasivam, S., Walker, P.S., 1999. The conflicting requirements of laxity and conformity in total knee replacement. Journal of biomechanics 32, 239-247.

Steinbruck, A., Milz, S., Woiczinski, M., Schroder, C., Utzschneider, S., Jansson, V., Fottner, A., 2011. Anatomie und Biomechanik des Patellofemoralgelenks. Physiologische Gegebenheiten und Veranderungen nach Implantation einer Knietotalendoprothese. Der Orthopade 40, 848, 850-2, 854.

Verjans, M., Asseln, M., Radermacher, K., 2016. Rapid prototyping of replica knee implants for in vitro testing. Current Directions in Biomedical Engineering 2.

\section{Disclosures}

This work has been supported in parts by Conformis, Inc., Bedford, USA. 